\title{
Experiences with QuickCheck: Testing the Hard Stuff and Staying Sane
}

\author{
John Hughes \\ Chalmers University/Quviq AB, Göteborg, Sweden
}

\begin{abstract}
This is not a typical scientific paper. It does not present a new method, with careful experiments to evaluate it, and detailed references to related work. Rather, it recounts some of my experiences over the last 15 years, working with QuickCheck, and its purpose is as much to entertain as to inform.

QuickCheck is a random testing tool that Koen Claessen and I invented, which has since become the testing tool of choice in the Haskell community. In 2006 I co-founded Quviq, to develop and market an Erlang version, which we have since applied for a wide variety of customers, encountering many fascinating testing problems as a result.

This paper introduces Quviq QuickCheck, and in particular the extensions made for testing stateful code, via a toy example in C. It goes on to describe the largest QuickCheck project to date, which developed acceptance tests for AUTOSAR C code on behalf of Volvo Cars. Finally it explains a race detection method that nailed a notorious bug plaguing Klarna, northern Europe's market leader in invoicing systems for e-commerce. Together, these examples give a reasonable overview of the way QuickCheck has been used in industrial practice.
\end{abstract}

\section{Introduction}

Here's an interesting little experiment. Try asking a room full of software developers, "who really, really loves testing?" Very, very few will raise their hands. For whatever reason, most developers see testing as more of a chore than a pleasure; few go to work in the morning raring to write some test cases. And yet, testing is a vital part of software development! Why should it be so unpopular?

To understand why, imagine writing a suite of unit tests for software with, say, $n$ different features. Probably you will write $3-4$ test cases per feature. This is perfectly manageable - it's a linear amount of work. But, we all know you will not find all of your bugs that way, because some bugs can only be triggered by a pair of features interacting. Now, you could go on to write test cases for every pair of features - but this is a quadratic amount of work, which is much less appealing. And even if you do so, you will still not find all of your bugs - some bugs only appear when three features interact! Testing for all of these would involve $O\left(n^{3}\right)$ work, which is starting to sound very unappealing indeed - and this is before we even start to consider race conditions, which by definition involve at least two 
index, incrementing the index afterwards modulo the size. Finally, size returns the number of elements currently in the queue, by taking the difference of the input and the output indices, modulo the size. The code is straightforward, and obviously correct - it's just a simple example to give us something to test.

Quviq QuickCheck provides a mechanism for testing C code directly from Erlang. In the Erlang shell, we can call

1> eqc_c:start $(q)$.

which compiles q.c (containing the code from Figure 1), and makes the functions in it callable from Erlang. This is done by parsing the $\mathrm{C}$ code to extract the types and functions it contains, generating a server that calls the $\mathrm{C}$ functions on demand, generating client code in Erlang that passes a function and arguments to the server and collects the result, and then running the server in a separate OS process so that buggy $\mathrm{C}$ code cannot interfere with the OS process running QuickCheck. The result is a safe test environment for $\mathrm{C}$ functions that might behave arbitrarily badly.

We can now perform simple tests to verify that the code is working as it should:

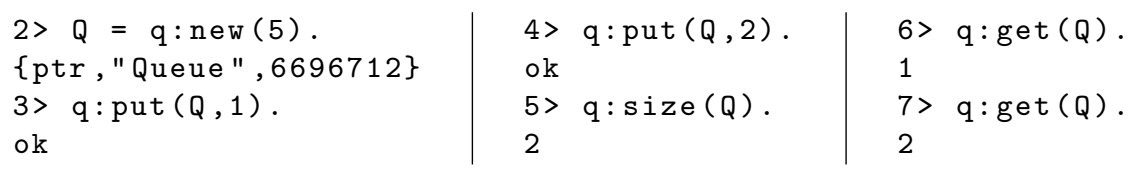

We create a queue with space for 5 elements, binding the pointer returned to Q. Then we put 1 and 2 into the queue, test size, and take the elements out of the queue again. All the results are as expected. We can even continue the test:

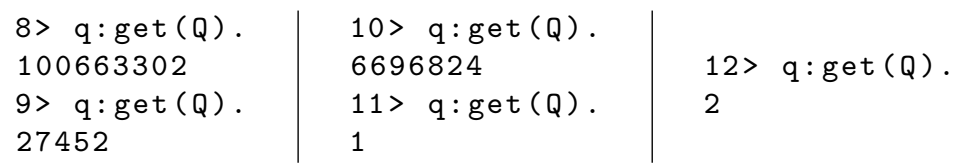

which returns the contents of uninitialized memory, as expected, until we reach the values 1 and 2 again. We really are running $\mathrm{C}$ code, which is just as unsafe as ever, and the queue implementation does not - and is not intended to-perform any error checking. If you abuse it by removing elements from an empty queue, you will get exactly what you deserve.

\section{Testing with QuickCheck}

Like much of the code Quviq works with, this example is stateful. We test stateful systems by generating sequences of calls to the API under test, just like the test cases that developers write by hand. But we also model the state of the system abstractly, and define model state transitions for each operation in the API. Using these state transition functions, we compute the model state at every point in a test. We define postconditions for each API call, relating the actual result of the call to the model state - see Figure 2. A test passes if all postconditions hold. 


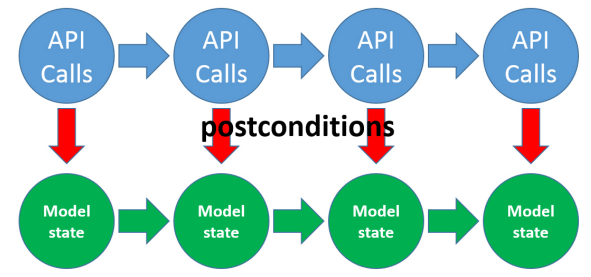

Fig. 2. Adjudging a test based on a state machine model.

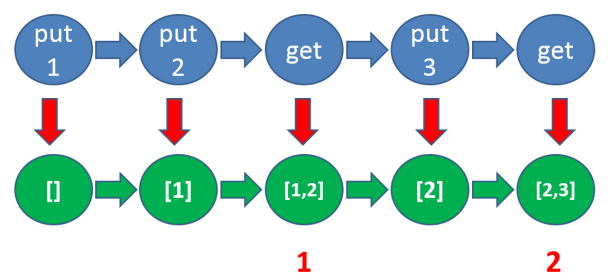

Fig. 3. A sample test case for the queue.

While the code we test can be in any programming language (such as $\mathrm{C}$ ), the model - the state transition functions, postconditions, etc.-is defined in Erlang.

In this case, we might model the state of the queue by a list of the integers that should be in it; with this model, a sample test case might appear as in Figure 3. Here we start with an empty queue (modelled by the empty list []), each put operation appends an element to the model state, each get operation removes an element from the state, and get's postcondition checks that the actual result (in red) was the first element of the model state before the call.

We will not present all the code of the model here, but to give its flavour, we present the specification of get:

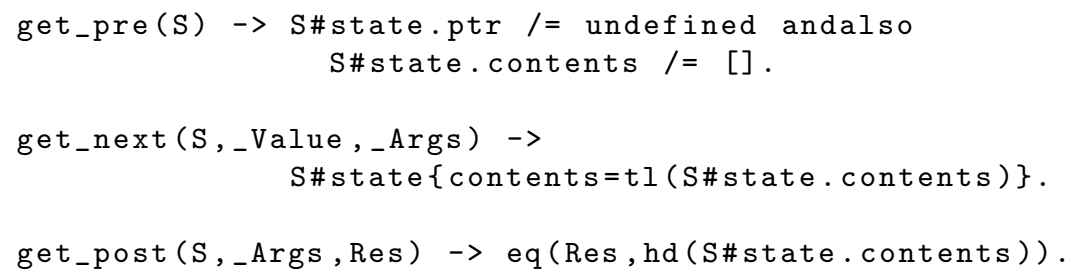

Here the model state $\mathbf{S}$ is actually an Erlang record (state) with fields ptr (pointer to the queue), contents (expected list of elements), and size (maximum size). get_pre is the precondition: get may only be called if the ptr is defined (i.e. new has been called), and the queue is non-empty. get_next is the state transition function: it replaces the list of elements in the model state by its tail. get_post is the postcondition, which checks that the actual result of get, Res, is equal to the first element of the expected contents. QuickCheck recognises these functions by virtue of their names (get followed by a particular suffix), and uses them to generate and run random tests. The property tested is: if all operation preconditions hold during a test run, then so do the postconditions. 
Tests are run by invoking QuickCheck from the Erlang shell, giving this property as a parameter. In this case, the tests fail almost immediately:

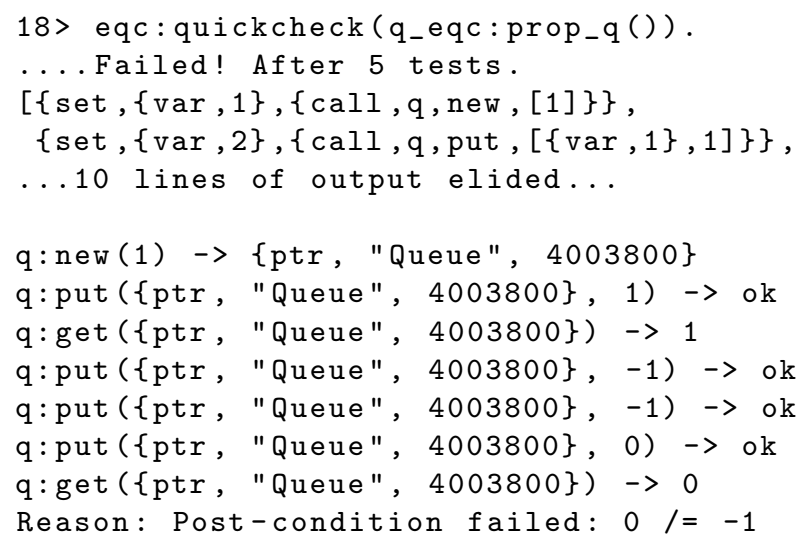

After four successful tests (represented by a '.' in the output), QuickCheck generated a failing example, which appears next in a symbolic form-but in this paper, we focus on the pretty-printed results that follow it (beginning with the line $q$ :new (1) $\rightarrow$....). These show the calls made and results returned - and clearly, something is wrong, because the postcondition of the last call failed. 0 , the actual result of the call, was not equal to -1 , the expected value.

Like most randomly generated failing tests, this one contains irrelevant calls as well as those few that actually provoked the failure. So QuickCheck then shrinks the test case to a simpler one, searching for the smallest similar test that also fails. In this case,the output continues:

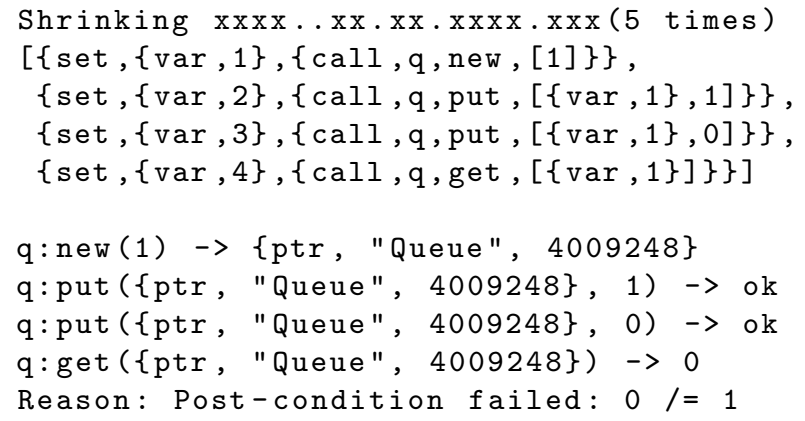

During shrinking, each '.' represents a smaller test that also failed-progress towards the minimal failing test - while ' $x$ ' represents a smaller test that passed. Shrinking not only removes unnecessary calls from the test case, but also simplifies arguments where possible - in particular, -1 no longer appears in the test.

While randomly generated failing tests vary widely, the result after shrinking is very consistent - always either the test case above, or this very similar one:

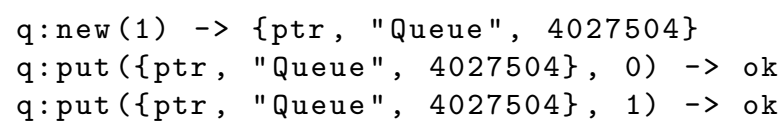


q: get (\{ptr, "Queue", 4027504\}) $\rightarrow 1$

Reason: Post-condition failed: $1 /=0$

\section{Debugging the model}

But why does the test above fail? Inspecting the results, we see that first we create a queue with space for one element, then we put a 0 into it, then we put a 1 into it - and at this point, the queue should contain [0,1] - then finally we get the first element, which should be 0 , but was actually 1 ! Clearly this is wrong!

But why did the $\mathrm{C}$ code return 1? Tracing through the example, we see that we allocated a buffer large enough to hold one integer, and then put two integers into it! Since we implemented a circular buffer, then the second value (1) overwrites the first (0). This is why get returns 1 - the data has been corrupted by putting too many elements into the queue.

This really is a minimal test case for this error: not only must we perform two puts and a get, but we must put two different values - otherwise we overwrite a value with an equal one, and the error is not detectable. This is why the values put are 0 and 1 - one of them is arbitrary, so can shrink to 0 , while the other must be different. The simplest value different from 0 is 1 , so this is the value we see. In retrospect, it was very informative that we did not see two zeroes - this told us immediately that if we were to put two zeroes, then the test would pass.

So where is the error? Recall that the code is not intended to behave sensibly when abused - and putting two elements into a queue of size one is surely abuse. Arguably, there is nothing wrong with the code - this is a bad test. This means that the fault is not in the implementation, but in the model. Inspecting the precondition of put in the model we have been using so far, we find

put_pre(S) $\rightarrow$ S\#state.ptr /= undefined.

This allows tests to call put at any time, as long as the queue pointer is defined. We should of course only allow calls of put if the queue is not already full:

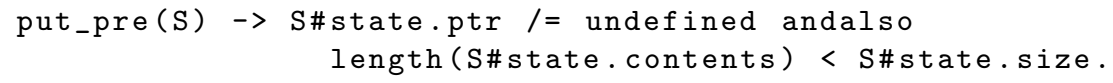

With this change to the precondition, we can repeat the last failed test, and see

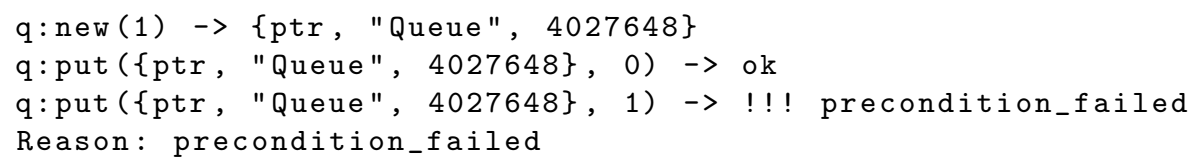

It still fails, but at an earlier point and for a different reason - the second call to put can no longer be made, because its precondition is not satisfied.

Random tests now pass, and we see satisfying output from QuickCheck:

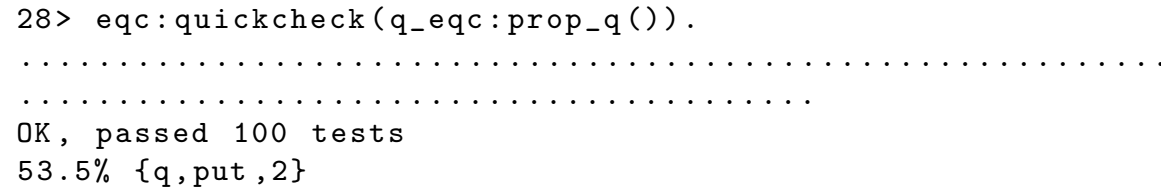


$40.2 \%\{q$, get, 1$\}$

$6.3 \%\{q$, new, 1$\}$

We usually collect statistics as tests are run; in this case we collect the names of the functions called $(\{q$, new, 1$\}$ represents $q$ : new $/ 1$, the new function with 1 argument in module q, and so on). Over $50 \%$ of the calls tested were to put, around $40 \%$ were to get - which is not so surprising, since given our preconditions, we cannot call get unless there is a corresponding call to put. However, these statistics reveal that we have not tested the size function at all! There is a simple reason for this - for simplicity, we omitted it from the model.

\section{Debugging the code}

It is easy to model the behaviour of size as well, but with this extension to the model, tests fail immediately. The shrunk example is this:

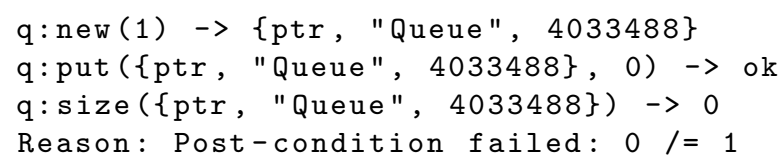

We create a queue with room for one element, put an element into it, then ask how many there are - which of course should be 1 , but size returns 0 ! This time the model is not at fault - the $\mathrm{C}$ code is simply returning the wrong answer.

Using this example we can trace through the code in Figure 1 to find the problem. new (1) initializes $q^{->}>$size to 1 . size returns

$$
(q->i n p-q->o u t p) \% q->s i z e
$$

But $q^{->}>$size is 1 , and any value modulo 1 is zero! It follows that size always returns 0 when the queue size is 1 , no matter how many times we call put. Likewise, put and get increment their respective indices modulo 1, so these indices are also always zero. Putting an element into the queue does not change its state - a full queue looks exactly the same as an empty one! This is the real problem, and it is not limited to queues of size one. If we create a queue of size $n$, and put $n$ items into it, then the same thing happens - the input index wraps around back to zero, and a full queue looks the same as an empty one. Our representation of queues is inadequate - it cannot distinguish full from empty.

How to fix this? There are several possibilities - we could, for example, add a boolean to the Queue struct to distinguish full queues, but this would lead to special cases throughout the code. Much nicer is to modify the new function so that, when asked to create a queue with space for $n$ elements, we actually create one with space for $n+1$ elements!

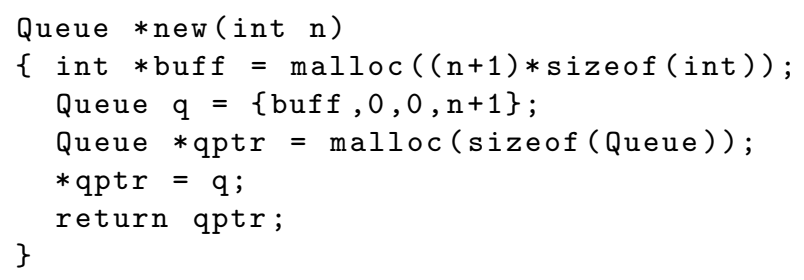


Let this be our secret. Now, the only way for a caller to witness the 'bug' is by putting $n+1$ elements into a queue with room for $n$-violating the precondition of put, which means the problem is their fault! We successfully avoid blame, and can go home happy.

Sure enough, with this change then repeating the last test succeeds:

32> eqc: check (q_eqc:prop_q()).

OK, passed the test.

However, running random tests provokes another failure, which always shrinks to the same test case:

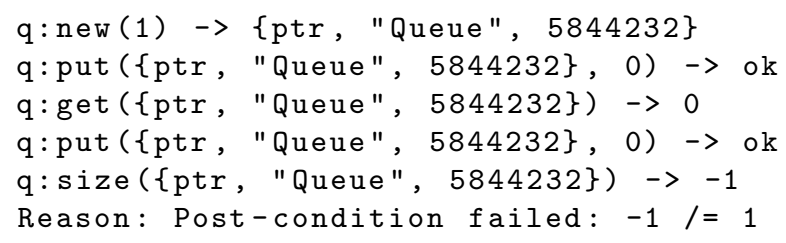

We create a queue of size one (that's really two!), and put a zero into it- - now it's full. We get the zero - and now it's empty, so we can put another zero in. Now the queue should contain one zero... but when we ask the size, then -1 is returned! This is clearly a bug in the $\mathrm{C}$ code - the size should never be negative.

Tracing this example through the code in Figure 1, we see that we really created a queue with a q->size of 2 , then called put twice, so the input index q->inp wraps around back to zero. We called get once, so q->outp is 1 , and in size, (q->inp - q->outp) \% q->size computes $(-1) \% 2$-but $(-1) \bmod 2$ is +1 , as we all learned in school. Or is it? Trying it in Erlang:

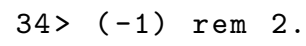

In Erlang, at least, rem is not the modulo operator - it computes the remainder after integer division, rounding towards zero. Since (-1) div 2 is zero, then $(-1)$ rem 2 must be -1 . The same is true in C.

With this in mind, we see that size returns a negative result whenever q->inp has wrapped around, but q->outp has not-whenever

$$
q->\text { inp - q->outp }
$$

is negative. To fix the bug, we must ensure that this expression is never negative, and a simple way to do so is to apply abs to it. We modify size as follows,

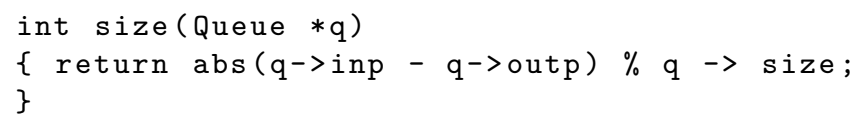

and repeat the last test:

36> eqc: check (q_eqc:prop_q ()).

OK, passed the test. 
Of course it passes, as we would expect.

Now, notice what we did. We found a subtle bug in our implementation. We created a test case that provoked the bug. We fixed the code, and our test now passes. All our tests are green! So... we're done, right?

In practice, many developers would be satisfied at this point. But let us just run a few more random tests... we find they fail immediately!

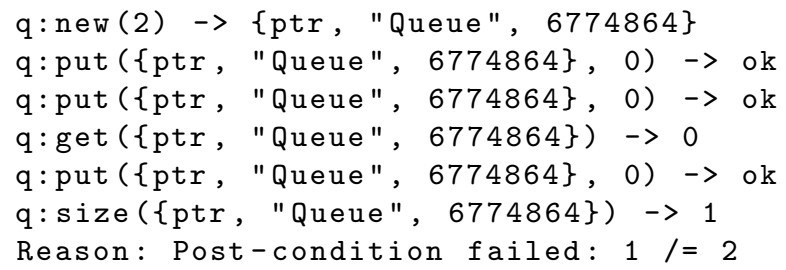

What's going on here? First, we create a queue of size two. Progress! The code now works - for queues of size one. We put three items into the queue, and remove one. Now there should be two items in the queue, but size returns 1 .

This is actually very similar to the example we just fixed. The queue is size two (really three!), so calling put three times makes the input index wrap around back to zero. We called get once, so the output index is one. The correct answer for size is thus $(-1) \bmod 3$, which is 2 , but we computed abs $(-1) \% 2$, which is 1 . Taking the absolute value was the wrong thing to do; it worked for the first test case we considered, but not in general.

A correct implementation must ensure that the operand of \% is non-negative, without changing its value modulo $\mathrm{q}^{->}>\mathrm{size}$. We can do this by adding $\mathrm{q}^{->} \mathrm{size}$ to it, instead of taking the absolute value:

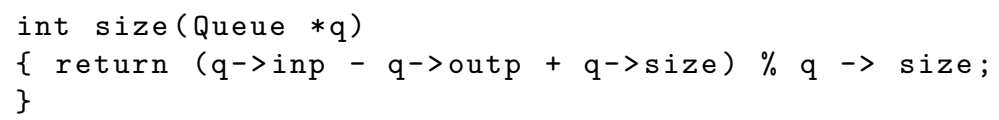

When we make this change, then this last failing test - and all the others we can generate - pass. The code is correct at last.

\section{Lessons from the queue example}

What can we learn from this? Three lessons that apply in general:

- The same property can find many different bugs. This is the whole point of test case generation: one model can provoke a wide variety of bugs.

- Errors are often in the model, rather than the code. Calling something a specification does not make it right! QuickCheck finds discrepancies between the model and the code; it is up to the developer to decide which is correct.

- Minimal failing test cases make debugging easy. They are often much smaller than the first failing one found. We think of shrinking as "extracting the signal from the noise". Random tests contain a great deal of junk - that is their purpose! Junk provokes unexpected behaviour and tests scenarios that the developer would never think of. But tests usually fail because of just a few features of the test case. Debugging a test that is $90 \%$ irrelevant is a 
nightmare; presenting the developer with a test where every part is known to be relevant to the failure, simplifies the debugging task enormously.

\section{Property-based testing}

I referred several times above to the "property" that is being tested, namely that "if all operation preconditions hold during a test run, then so do the postconditions". Properties are fundamental to QuickCheck: indeed, QuickCheck always tests a property, by generating suitable test cases and checking that the property holds in each case. Properties are often expressed in terms of a stateful modelas in this section - but this is not always the case, and even for the same model, the properties tested may differ. Since properties are fundamental here, we call the general approach "property-based testing", rather than "model-based testing" which is a better-established term.

\section{The Volvo Project}

So far, we only considered a toy example - the reader could be forgiven for wondering if the method really scales. After all, in this example, the specification was actually larger than the code! QuickCheck would not be useful if this were true in general. So, let us summarize the results of the largest QuickCheck project so far-acceptance testing of AUTOSAR Basic Software for Volvo Cars (Arts, Hughes, Norell, and Svensson, 2015).

Modern cars contain 50-100 processors, and many manufacturers base their software on the AUTOSAR standard. Among other things, this defines the 'Basic Software', which runs on every processor in the car. It consists of an Ethernet stack, a CAN-bus stack (the CAN bus, or "Controller Area Network", is heavily used in vehicles), a couple of other protocol stacks, a routing component, and a diagnostics cluster (which records fault codes as you drive). The basic software is made up of many modules, whose behaviour is specified in detail, but there are a number of suppliers offering competing implementations. Usually a car integrates basic software from several different suppliers - which means that if they do not follow the standard, then there is a risk of system integration problems. It is not even easy to tell, if two processors cannot talk to each other, which of the suppliers is at fault!

Volvo Cars wanted to test their suppliers code for conformance with the standard, and funded Quviq to develop QuickCheck models for this purpose.

Most errors we found are confidential, but we can describe one involving the CAN bus stack, which we tested using a mocked hardware bus driver. Now, every message sent on the CAN bus has a CAN identifier, or message type, which also serves as the priority of the message - the smaller the CAN identifier, the higher the priority. QuickCheck found a failing test with the following form:

- Send a message with CAN identifier 1 (and check that the hardware driver is invoked to put it onto the bus).

- Send a message with CAN identifier 2 (which should not be passed to the driver yet, because the bus is busy). 
- Send a message with CAN identifier 3 (which should also not be sent yet).

- Confirm transmission of message 1 by the bus (and check that the driver is now invoked to send message 2).

Of course, the bug was that the stack sent the message with identifier 3 instead.

This $i s$ the smallest test case that can provoke the bug. We must first send a message, making the bus busy, and then send two more with different priorities, which are both queued. Then we must release the bus, and observe that the wrong message is taken from the queue. This is just what the test does.

The source of the bug was this: the original CAN standard allowed only 11 bits for the message identifier, but today 2048 different message types is no longer enough, so the current standard also allows an 'extended CAN id' of 29 bits. Both kinds have the same meaning - in particular, the priority does not depend on the encoding - but when the stack sends a message, it must know which format to use. This particular stack stored both kinds of identifier in the same unsigned integer, but used bit 31 to indicate an extended identifier. In this test, message number 2 used an extended CAN identifier, while message number 3 used the original format. Of course, when comparing stored message identifiers in order to decide which message to send, it is essential to mask off the top bit... which the developer forgot to do in this case. So the second message was treated as priority $2^{31}+2$, and was not chosen for sending next.

The actual fault is a tricky mistake in low-level $\mathrm{C}$ code - nevertheless, it can be provoked with a short sequence of API calls, and we can find this sequence by generating a random one and shrinking it.

The fault is also potentially serious. Those priorities are there for a reason. Almost everything in the car can talk on the CAN bus - the brakes, the stereo.... Here's a tip: don't adjust the volume during emergency braking!

For this project we read around 3,000 pages of PDFs (the standards documents). We formalized the specification in 20,000 lines of QuickCheck code. We used it to test a million lines of $\mathrm{C}$ code in total, from 6 different suppliers, finding more than 200 problems - of which well over 100 were ambiguities or inconsistencies in the standard itself! In the few cases where we could compare our test code to traditional test suites (in TTCN3), our code was an order of magnitude smaller-but tests more.

So, does the method scale? Yes, it scales.

\section{Debugging a database}

The last story (Hughes and Bolinder, 2011) begins with a message to the Erlang mailing list, from Tobbe Törnqvist at Klarna.

"We know there is a lurking bug somewhere in the dets code. We have got 'bad object' and 'premature eof' every other month the last year. We have not been able to track the bug down since the dets files is repaired automatically next time it is opened." 
The context: Klarna was a Swedish start-up offering invoicing services to web shops $^{1}$. They built their product in Erlang, and stored their data in Mnesia, the database packaged with the Erlang distribution. Mnesia provides transactions, distribution, replication, and so on, but needs a back end to actually store the data. The back end for storage on disk is dets, which stores tuples in files. This problem piqued my interest - it was quite notorious in the Erlang community, and sounded as though it might involve a race condition.

To explain how QuickCheck tests for race conditions, let us consider a much simpler example: a ticket dispenser such as one finds at delicatessen counters, which issues each customer with a ticket indicating their place in the queue. We might model such a dispenser in Erlang by two functions, take_ticket() and-because the roll of tickets needs to be replaced sometimes-reset(). A simple unit test for these functions might look as follows:

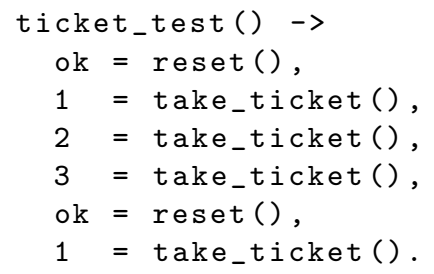

Here we use Erlang pattern matching to compare the result of each call with its expected value (ok being an atom, a constant, in Erlang - conventionally used as the result of a function with no interesting result). This test case is typical of test cases written in industry: we make a sequence of calls, and compare the results to expected values.

It is easy to create a QuickCheck state machine for testing the dispenser also. We can model its state as a single integer, which is set to 0 by reset(), incremented by take_ticket(), and used in the postcondition of take_ticket to check the result.

However, running sequential tests of the ticket dispenser is not enough: the whole point of the system is to regulate the flow of many concurrent customers to one delicatessen counter. If we do not also run parallel tests of the API, then we are failing to test an essential part of its behaviour.

Now, consider one simple parallel test:

\begin{tabular}{|c|c|}
\hline \multicolumn{2}{|c|}{ reset () } \\
\hline $\begin{array}{l}\text { take_ticket () } \\
\text { take_ticket () }\end{array}$ & take_ticket () \\
\hline
\end{tabular}

which represents first resetting the dispenser, then two customers taking tickets in parallel - the left customer taking two tickets, the right customer taking one. What are the expected results? Well, the left customer might get tickets \#1 and $\# 2$, and the right customer ticket \#3. Or, the left customer might get tickets $\# 1$ and \#3, while the right customer gets ticket \#2! But if both customers get ticket number \#1, then the test has failed.

\footnotetext{
1 They have since grown to well over 1,000 people, serving 8 countries and counting.
} 
This poses a problem for the traditional way of writing test cases, because we cannot predict the expected results. In this case, we could in principle compute all three possibilities (in which the right customer gets ticket number \#1, \#2 or \#3), and compare the actual results against all three - but this approach does not scale at all. Consider this only slightly larger test:

\begin{tabular}{|l||l||l|}
\hline \multicolumn{2}{|c|}{$\operatorname{reset}($ ) } \\
\hline take_ticket() & take_ticket () & reset() \\
take_ticket() & take_ticket () & \\
\hline
\end{tabular}

This test has thirty possible correct outcomes! No developer in their right mind would try to enumerate all of these - and anyone who tried would surely get at least one wrong. The only practical way to decide if a test such as this has passed or failed, is via a property that distinguishes correct from wrong results. Parallel testing is a 'killer app' for property-based testing.

The way that QuickCheck decides whether a test like this has passed is by searching for any interleaving of the calls which makes their results match the model. If there is such an interleaving, then the test passes - but if there is no such interleaving, then it has definitely failed. Here we are using the same model as we used for sequential testing, to test a different property of the implementation - namely, serializability of the operations.

Testing a buggy version of the dispenser, QuickCheck immediately reports:

Parallel:

1. dispenser:take_ticket() $-->1$

2. dispenser:take_ticket() $-->1$

Result: no_possible_interleaving

That is, two parallel calls of take_ticket can both return 1-but no sequence of two calls can return these results. In this case, there is a blatant bug in the code: take_ticket ( ) reads and then writes a global variable containing the next ticket number, with no synchronization at all - so no wonder there is a race condition. But the point is that QuickCheck finds the bug very fast, and shrinks it to this minimal example very reliably indeed.

Returning to dets, it stores tuples of the form $\left\{\mathrm{Key}, \mathrm{Val}_{1}, \mathrm{Val}_{2} \ldots\right\}$ in a file, and its API is unsurprising. For example,

- insert (Table,List) inserts a list of tuples into a table,

- delete(Table,Key) deletes all tuples with the given key from the table, and

- insert_new (Table,List) is like insert, unless one of the keys to be inserted is already in the table, in which case it is a no-op.

These operations are all guaranteed to be atomic.

A state machine modelling dets is easy to construct - it is almost enough just to model the table by a list of the tuples that should be in it. My model of the core of the dets API is less than 100 lines of code - which compares well to the implementation, over 6,000 lines of code spread over four modules, maintaining hash tables on the disk, supporting an old format for the files, and so on. Thus, although dets is quite complex, its intended behaviour is simple. 
I first tested the model thoroughly by running tens of thousands of sequential tests. This ensures that the model and the code are consistent - and it did turn up a few surprises. There is no point running parallel tests of a system, if inconsistencies can already be found using sequential tests. (I assumed that dets behaves correctly in the sequential case -it is mature and well-tested code, after all - so any inconsistencies indicate a misunderstanding of the intended behaviour, i.e. a bug in the model).

Once the model was correct, I began to run parallel tests. This only required writing a few lines of code, because we reuse the same model for sequential and parallel testing. Almost immediately, a real bug appeared:

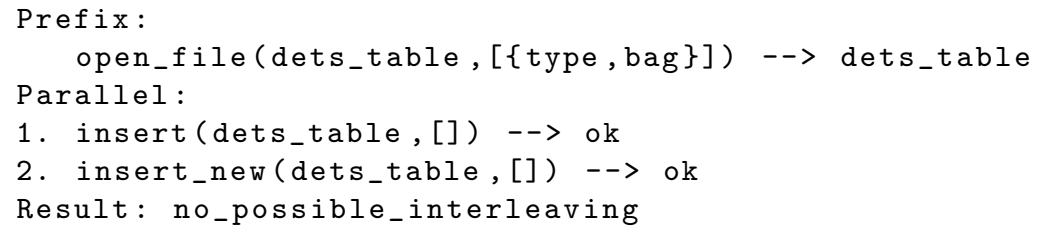

In the sequential prefix of the test, we open the dets file, then in parallel call insert and insert_new. Both insert an empty list of tuples - so they are both no-ops - and they both return ok! At first sight this looks reasonable - but the documentation for insert_new says it should return a boolean! Indeed, in tens of thousands of sequential tests, insert_new returned true or false every timenow, suddenly, it returned ok. Strange!

Another run of QuickCheck reported a second bug:

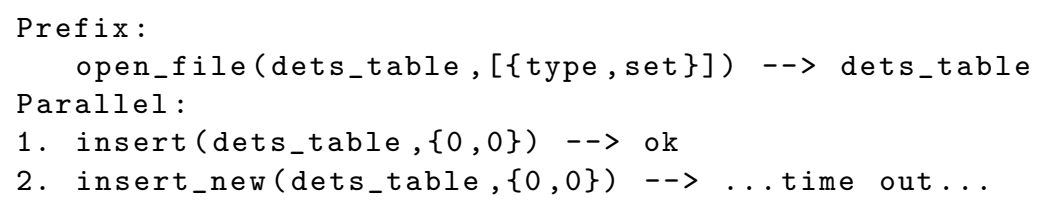

In this case, both insert and insert_new try to insert the same tuple, which means that insert_new could succeed or fail... but it should not time out, and we should not see the message

$=$ ERROR REPORT $====4-0 c t-2010:: 17: 08: 21===$

** dets: Bug was found when accessing table dets_table

At this point I disabled the testing of insert_new, which seemed not work in parallel, and discovered a third bug:

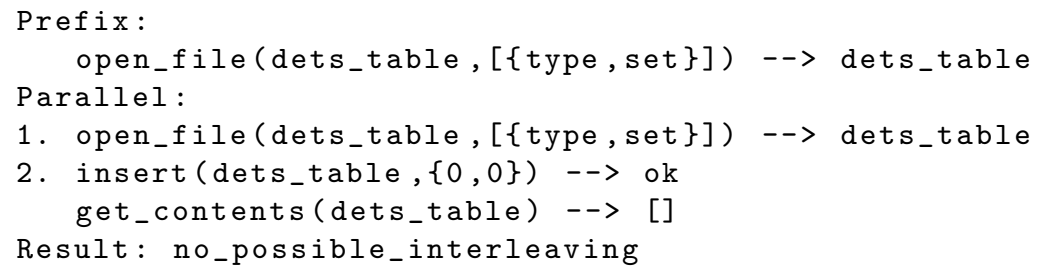

Here we first open the file, and then in parallel open it again, and insert a tuple, then fetch the entire contents of the table. The test fails because process 
2 sees an empty table, even though it just inserted a tuple! It may seem a little suspicious to open the table twice, but it is not at all - Erlang is designed for highly concurrent applications, so we expect many processes to be using the table at the same time, and all of them need to make sure it is open.

I reported these bugs to Hans Bolinder at Ericsson, who was responsible for the dets code, and next day he sent me a thank you and a fixed version. However, he thought these were probably not the bugs that were plaguing Klarna, because the symptoms were different. At Klarna, the file was being corrupted. Hans gave me one line of code that could check for corruption, and I added it to the test to check that, after executing a parallel test, the file was not corrupt.

This time it took around 10 minutes of testing to find a bug:

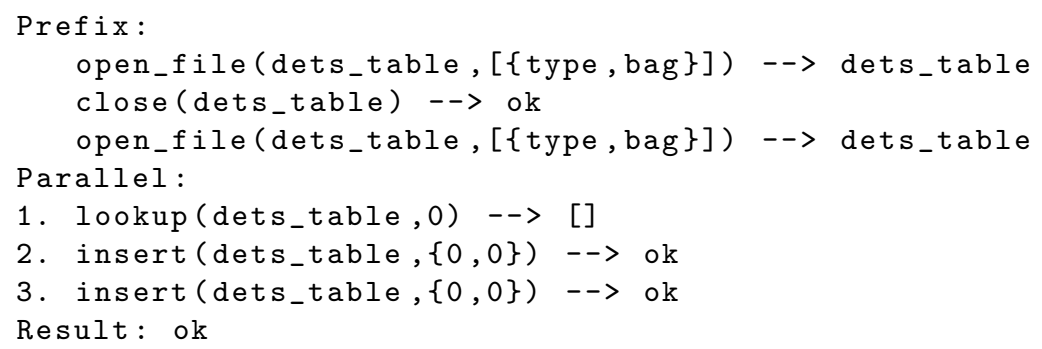

First we open, close, and open the file again, and then we do three things in parallel: a lookup of a key that is not found, and two insertions of the same tuple. All results are consistent with the model - but the corruption check encountered a 'premature eof'.

This is really the smallest test case that can provoke this error. It was initially hard to believe that the open-close-open sequence was really necessary. I manually removed the first open and close, and ran the smaller test case tens of thousands of times. It passed, every single time. Today I know why: the first call to open creates the file, while the second call opens an existing file. It's slightly different, and dets enters a slightly different state - and that state is key to provoking the bug. Three parallel operations are needed because the first makes the dets server busy, causing the next two to be queued up, and then (wrongly) dispatched in parallel by the server, once the first call is complete.

The final bug was found when preparing a talk on this experience soon afterwards, whlie rerunning the tests in order to copy the output onto slides. Again, after around ten minutes of testing, the following bug was found:

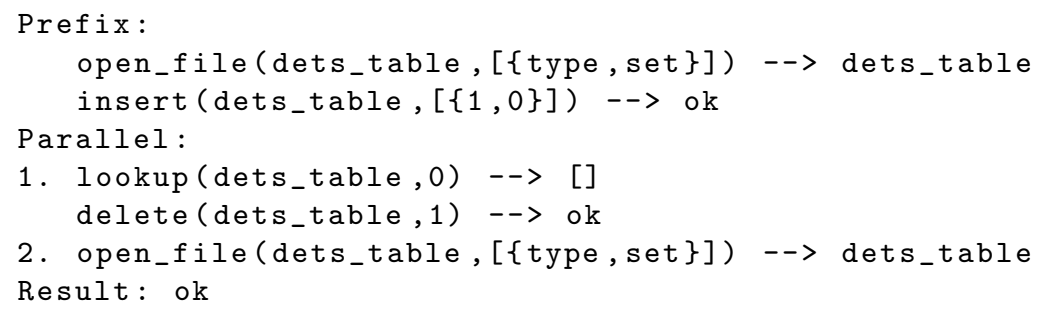

We open the file and insert a tuple, then, in parallel, reopen the file, and lookup a key not in the table, then delete the key that is. All the results are consistent 
with the model — but the corruption check encountered a 'bad object'. Recall the mailing list message:

"We know there is a lurking bug somewhere in the dets code. We have got 'bad object' and 'premature eof' every other month the last year."

So it seemed these might indeed be the Klarna bugs.

In each case, Hans Bolinder returned a fixed version of the code within a day of receiving the bug report - and at Klarna, where the problem had meanwhile begun to appear once a week, there has been only one 'bad object' error since the new code went into production... when a reading a file last written before the new code was installed.

Prior to this work Hans Bolinder had spent six weeks at Klarna hunting for the bug, and the best theory was that it struck when the file was around 1GB, and might be something to do with rehashing. Now we know the bugs could be provoked with a database with at most one record, using only 5-6 calls to the API. In each case, given the minimal failing test, it was less than a day's work to find and fix the bug. This really demonstrates the enormous value of shrinking - and shows just how hopeless it is to try to debug this kind of problem from failures that happen in production, where not only the five or six relevant things have occurred, but also millions of irrelevant others.

\section{In conclusion}

This paper recounts some of our experiences of using an advanced testing tool in real applications - its purpose is as much to entertain as to inform. Of course, there is a wealth of other work in the area, that this paper makes no attempt to cover-RANDOOP (Pacheco, Lahiri, Ernst, and Ball, 2007), DART (Godefroid, Klarlund, and Sen, 2005), and their successors would be good starting points for further reading.

I have also just touched the surface of the work that we have done. There was far more to the Volvo project than could be described here. We needed to develop a property-based approach to mocking (Svenningsson, Svensson, Smallbone, Arts, Norell, and Hughes, 2014). We needed to develop ways to cluster models of individual components into an integrated model of a subsystem, because AUTOSAR subsystems are typically implemented monolithically, but specified as a collection of interacting components. We found that QuickCheck's shrinking tended to isolate the same bug in each run - which is not ideal when generating a test report on a component with several different bugs - so we developed methods to direct testing away from already-known problems towards areas where unknown bugs might still lurk, and report all the bugs at once. We were asked "does your model cover this test case?", so we developed tools to answer such questions - and when the AUTOSAR consortium released six "official" test cases for the CAN bus stack, we used them to show that three of these test cases were not covered by the model, because they were not consistent with the standard (Gerdes, Hughes, Smallbone, and Wang, 2015). We were asked: "which requirements have you tested?", so we developed ways to collect requirements 
coverage during testing - leading us to question what testing a requirement even means (Arts and Hughes, 2016).

In other areas, we helped Basho test their no-SQL database, Riak, for the key property of eventual consistency - and found a bug (now fixed, of course) that was present, not only in Riak, but in the original Amazon paper (DeCandia, Hastorun, Jampani, Kakulapati, Lakshman, Pilchin, Sivasubramanian, Vosshall, and Vogels, 2007) that kicked off the no-SQL trend. Recently we tested Dropbox's file synchronization service, with some surprising results there too (Hughes, Pierce, Arts, and Norell, 2016). Both these applications were based in part on the state machine framework presented here, but added additional properties to express subtle correctness conditions.

Perhaps the most general lesson from all this experience is that formulating specifications is hard - and many developers struggle with it. It may often be easier to give examples of correct behaviour, than to define in general what correctness means. This should not come as a surprise, since mathematicians have used examples for centuries - but it does suggest that starting from a specification may not always be appropriate. Sometimes I am asked: "wouldn't it be great if we could just synthesize code from a specification?" It would not: we would just replace buggy code with buggy specifications. A key insight from using QuickCheck is that we find bugs by comparing two independent descriptions of the desired behaviour - the implementation, and the specification - and it is the inconsistencies between the two that reveal errors.

The need for a specification is the real weakness of property-based testingnot that we use testing, rather than static analysis or proof, to relate specifications and implementations. Testing works well enough! The real question is: how can we make specifications more useable, and easier to construct - for real systems with all their complex behaviours? There is much work to be done hereperhaps future tools will even help developers construct specifications from examples.

In any event, there are many fascinating problems waiting to be addressed. If this paper helps persuade the reader that testing is not a chore that must be done, but a fascinating research area in its own right, then it will have fulfilled its purpose.

And don't forget,

\section{DON'T WRITE TESTS, GENERATE THEM!}

\section{Acknowledgements}

The work described here was partially supported by the EU FP7 project "PROWESS", and by the Swedish Foundation for Strategic Research grant "RAWFP".

\section{References}

Arts, T., Hughes, J., Norell, U., Svensson, H.: Testing AUTOSAR software with

QuickCheck. In: Software Testing, Verification and Validation Workshops 
(ICSTW), 2015 IEEE Eighth International Conference on. pp. 1-4 (April 2015)

Arts, T., Hughes, J.: How well are your requirements tested? In: Briand, L., Khurshid, S. (eds.) International Conference on Software Testing, Verification and Validation (ICST). IEEE (April 2016), to appear

Arts, T., Hughes, J., Johansson, J., Wiger, U.: Testing Telecoms Software with Quviq QuickCheck. In: Proceedings of the 2006 ACM SIGPLAN Workshop on Erlang. pp. 2-10. ERLANG '06, ACM, New York, NY, USA (2006)

Claessen, K., Hughes, J.: QuickCheck: A Lightweight Tool for Random Testing of Haskell Programs. In: Proceedings of the Fifth ACM SIGPLAN International Conference on Functional Programming. pp. 268-279. ICFP '00, ACM, New York, NY, USA (2000)

DeCandia, G., Hastorun, D., Jampani, M., Kakulapati, G., Lakshman, A., Pilchin, A., Sivasubramanian, S., Vosshall, P., Vogels, W.: Dynamo: Amazon's Highly Available Key-value Store. In: Proceedings of Twenty-first ACM SIGOPS Symposium on Operating Systems Principles. pp. 205-220. SOSP '07, ACM, New York, NY, USA (2007)

Gerdes, A., Hughes, J., Smallbone, N., Wang, M.: Linking Unit Tests and Properties. In: Proceedings of the 14th ACM SIGPLAN Workshop on Erlang. pp. 19-26. Erlang 2015, ACM, New York, NY, USA (2015)

Godefroid, P., Klarlund, N., Sen, K.: DART: Directed Automated Random Testing. In: Proceedings of the 2005 ACM SIGPLAN Conference on Programming Language Design and Implementation. pp. 213-223. PLDI '05, ACM, New York, NY, USA (2005)

Hughes, J.: QuickCheck Testing for Fun and Profit. In: Proceedings of the 9th International Conference on Practical Aspects of Declarative Languages. pp. 1-32. PADL'07, Springer-Verlag, Berlin, Heidelberg (2007)

Hughes, J., Pierce, B., Arts, T., Norell, U.: Mysteries of Dropbox: PropertyBased Testing of a Distributed Synchronization Service. In: Briand, L., Khurshid, S. (eds.) International Conference on Software Testing, Verification and Validation (ICST). IEEE (April 2016), to appear

Hughes, J.M., Bolinder, H.: Testing a Database for Race Conditions with QuickCheck. In: Proceedings of the 10th ACM SIGPLAN Workshop on Erlang. pp. 72-77. Erlang '11, ACM, New York, NY, USA (2011)

Pacheco, C., Lahiri, S.K., Ernst, M.D., Ball, T.: Feedback-Directed Random Test Generation. In: Proceedings of the 29th International Conference on Software Engineering. pp. 75-84. ICSE '07, IEEE Computer Society, Washington, DC, USA (2007)

Svenningsson, J., Svensson, H., Smallbone, N., Arts, T., Norell, U., Hughes, J.: An Expressive Semantics of Mocking. In: Proceedings of the 17th International Conference on Fundamental Approaches to Software Engineering Volume 8411. pp. 385-399. Springer-Verlag New York, Inc., New York, NY, USA (2014) 